\title{
New Insights into the Investigation of Smoke Production Using a Cone Calorimeter
}

\author{
R. Sonnier* (1), Centre des Matériaux des Mines d'Alès (C2MA), 6, Avenue de \\ Clavières, 30319 Alès Cedex, France \\ H. Vahabi, Laboratoire MOPS E.A. 4423, Université de Lorraine, 57070 Metz, \\ France \\ C. Chivas-Joly, LNE, 29 Avenue Roger Hennequin, 78197 Trappes Cedex, \\ France
}

\begin{abstract}
Smoke release data from the cone calorimeter are often underused. They may provide additional information to better understand the fire reaction of polymers and the efficiency of flame retardants. A new method is proposed to investigate the smoke release in cone calorimeter tests and to correlate it to heat release, based on studies with pure and flame retarded polymers. Smoke release rate is plotted versus heat release rate and new parameters are pointed out. In particular, parameter A represents the smoke release per unit energy (in Joules) released. Its value increases when the carbon fraction and the aromaticity of a polymer increase. It can reach around $0.05 \mathrm{~m}^{2} / \mathrm{kJ}$ for epoxy resins but is null for well-known smoke-free polyoxymethylene $(\mathrm{POM})$. HRR threshold $\left(\mathrm{HRR}_{\mathrm{th}}\right)$ represents the critical heat release rate above which smoke release is measured. Its value is close to $100 \mathrm{~kW} / \mathrm{m}^{2}$ for polyolefins but decreases drastically for aromatic polymers. The approach developed in this study is potentially useful for assessing the smoke release of different materials for a heat release rate scenario chosen arbitrarily. The influence of two specific smoke suppressants and of two specific flame retardants on smoke release is also discussed and the proposed method allows for a better understanding of their role in smoke release.
\end{abstract}

Keywords: Cone calorimeter, Smoke production, Flame inhibitor, Smoke suppressant

\section{Introduction}

Statistics indicate that, in actual fires, the cause of fire fatalities is often attributed to smoke inhalation, but such fire fatalities typically result from fires that have grown to be very large (i.e. high heat release) $[1,2]$. One effect that is very important in fires is the lack of visibility, as caused by high smoke obscuration [3, 4]. In a number of regulations and specifications there are limits on the allowable smoke release. The concerns about smoke obscuration are of particular importance when

\footnotetext{
* Correspondence should be addressed to: R. Sonnier, E-mail: rodolphe.sonnier@mines-ales.fr
} 
escape (or rescue) is difficult, for example in public transport [5, 6]. Furthermore, there is a controversy about the benefits of flame retardants. Indeed, they tend to delay ignition and reduce heat release but they can also promote black (and toxic) smoke. Therefore it is important to properly compare the impact of different flame retardants on both heat release and smoke production. In this article we only consider smoke opacity.

One characteristic material property related to smoke is the smoke point. Smoke is due to soots which are produced in the lower part of the flame and are oxidized in its upper part. When soots are not fully oxidized due to excessively fast fuel production, smoke is released from the flame tip. The height of the flame (which is also related to the fuel flow rate) when smoke begins to be released is called smoke point [7]. Low smoke point corresponds to materials producing a high amount of soots during burning with laminar flame. The smoke point can easily be determined for gases or liquids but some authors have proposed methods to measure it for solid fuels [7, 8]. Even if this property is useful, it only corresponds to the critical fuel production rate above which smoke appears. Nevertheless, above this critical value, it is important to assess how smoke production increases with fuel production rate.

The most common small-scale tests used for assessing smoke obscuration are the closed smoke density chamber, where the smoke accumulates during the test [9]. However, smoke obscuration can also be assessed in flow-through systems and this is the way it is measured in the majority of large-scale (and even intermediatescale) fire tests. One example of a small-scale fire test which can be used to assess smoke obscuration in a flow-through system is the cone calorimeter. There is often no direct way to correlate smoke obscuration measured in a closed system with that measured in a flow-through system.

In the cone calorimeter, the air flow rate is normally set at $24 \mathrm{~L} / \mathrm{s}$, the combustion process is well-ventilated and the combustion efficiency is generally high.

It is of interest to analyze smoke release data from the cone calorimeter to understand whether it can shed light on the mechanism of thermal decomposition of materials or on some specific phenomena associated with the effects of flame retardants or smoke suppressants. Studies presenting results of cone calorimeter tests often show heat release data only. In some cases, when smoke release data are shown, it was found that the shape of the curve of smoke release rate (SRR or RSR, in $\left.\left(\mathrm{m}^{2} / \mathrm{s}\right) / \mathrm{m}^{2}\right)$ versus time is very close to that of heat release rate (HRR, in units of $\mathrm{kW} / \mathrm{m}^{2}$ ) versus time. For example compare HRR and SPR curves of various EVA composites studied by $\mathrm{Wu}$ et al. [10]. This occurs when there is a direct correlation between smoke production and the release of energy. $\mathrm{Xu}$ et al. have shown that the correlation between heat release rate and smoke production rate is more complex in some cases [11].

The objective of this work is to describe an original method to get a new insight in the mechanism of decomposition in the cone calorimeter by using smoke production data. The study used two series of common flame retardant additives to illustrate the usefulness of this method. The first set of additives involved two mineral fillers, beneficial as smoke suppressants, namely aluminum hydroxide (ATH) and magnesium hydroxide (MDH). The second series of additives involved 
flame inhibitor additives, namely a brominated flame retardant (TBBA-tetrabromobisphenol A) and a phosphorus-based flame retardant (DOPO-9,10-dihydro-9oxy-10-phosphaphenanthrene-10-oxide).

\section{Experimental}

Both flame retarded and non-flame retarded polymers were tested. All the virgin polymers were commercially available. The flame retardants were incorporated into the polymers using a twin screw extruder (Clextral BC21). Four series of tests were conducted.

- The first set of samples (series A) involved non-flame retarded polymers tested in the cone calorimeter at a heat flux of $35 \mathrm{~kW} / \mathrm{m}^{2}$, with the sample positioned directly in the sample holder without placing a refractory fiber blanket under the sample. The distance between the top of the sample and the bottom of the radiant cone heater was $60 \mathrm{~mm}$. The objective of this (non-standard) set-up was to limit the rate of increase of HRR. It was also intended to aid in the measurement of parameter A (described below).

- Series B involved gathering data on several non-flame retarded polymers tested under various standard conditions (i.e. with a refractory fiber blanket). The tests were performed over several years for different projects and the results provide a large database. Most samples were tested at $35 \mathrm{~kW} / \mathrm{m}^{2}$. When heat flux is different, it is specified in Table 1). Note that the data points are too scarce to conveniently establish a general tendency about the influence of heat flux on the calculated parameters.

- Series C involved ethylene-vinyl acetate copolymer (EVA) without flame retardant or smoke suppressant additives and the same EVA containing various amounts $(20,40$ or $60 \mathrm{wt} \%)$ of the mineral fillers. The ATH used was Martinal LEO 104 from Martinswerk Gmbh and the MDH used was Magnifin H10 from Martinswerk Gmbh. The tests were conducted under standard conditions with the refractory fiber blanket and at a heat flux of $35 \mathrm{~kW} / \mathrm{m}^{2}$.

- Series D involved tests on polyethylene (PE) and polypropylene (PP), without and with added flame retardants, at an additive level of $10 \mathrm{wt} \%$. The flame retardants used were 9,10-dihydro-9-oxy-10-phosphaphenanthrene-10-oxide (DOPO, from TCI Europe) and Tetrabromobisphenol A (TBBPA). The heat flux was set at $35 \mathrm{~kW} / \mathrm{m}^{2}$. A grid was used to maintain the thickness of the samples at $2 \mathrm{~mm}$. Test conditions were similar to those in series A (no refractory fiber blanket, distance between top of sample and radiant cone heater set at $60 \mathrm{~mm}$ ).

Series C and D were prepared with the sole objective of studying smoke suppressants and flame inhibitors using the approach developed here. Mineral fillers are often used as smoke suppressants and DOPO and TBBPA are well known as flame inhibitors. It is not our objective to discuss in detail the fire performance of these materials. 


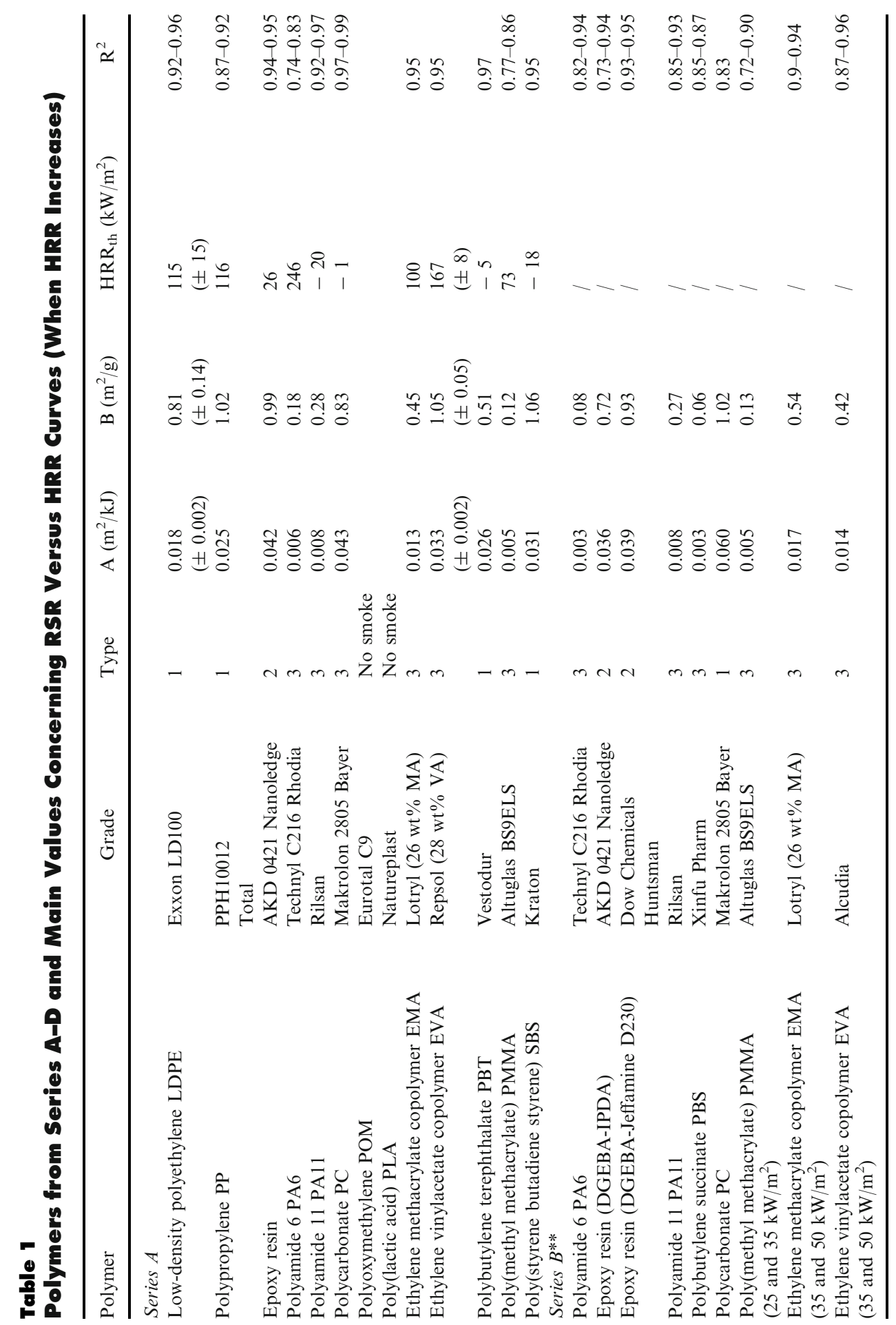




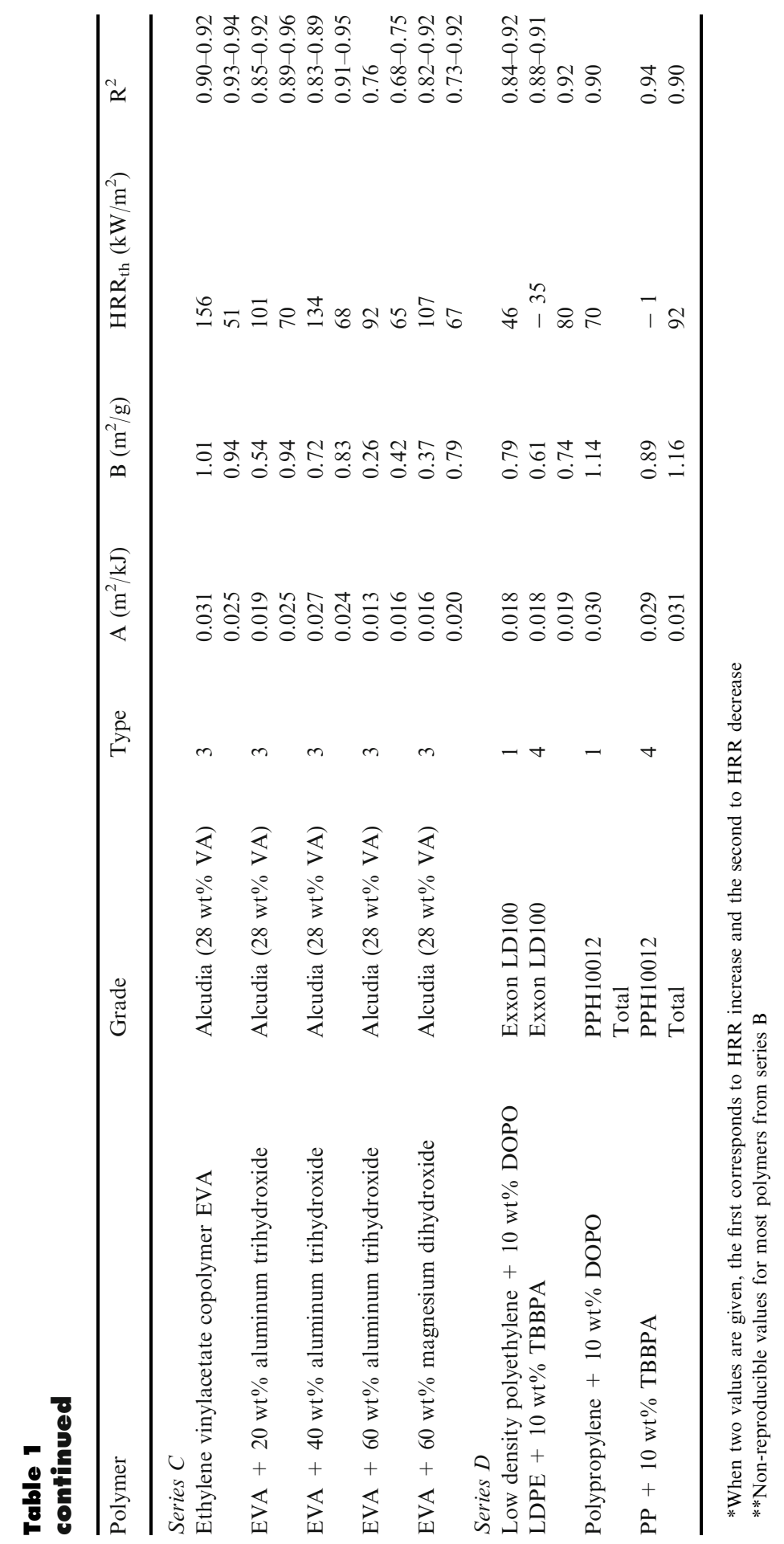


Each formulation was tested in either duplicate or triplicate (using the standard exposed surface of $10 \times 10 \mathrm{~cm}^{2}$ and a sample thickness of $4 \mathrm{~mm}$ for series A, B and $\mathrm{C}$, but $2 \mathrm{~mm}$ for series D), using a cone calorimeter from Fire Testing Technology. The procedure followed ISO 5660 (except for the use of the refractory fiber blanket and the distance to the sample for series A and D). The air flow rate was set at $24 \mathrm{~L} / \mathrm{s}$, and the standard spark igniter was used. A total of 84 tests were performed to validate this new method.

The emission of $\mathrm{HBr}$ from the decomposition of the brominated materials was continuously recorded by coupling the cone calorimeter to an Antaris IGS analyzer with an MCT detector from Thermo Scientific Company. Both the IR spectrometer and the transfer line were heated to $160^{\circ} \mathrm{C}$ to minimize the condensation of high boiling point effluents.

All formulations are listed in Table 1 together with main results obtained.

\section{Methods}

Cone calorimeter standard tests provide three different measurements of smoke. The smoke release rate (SRR or RSR, in $\left(\mathrm{m}^{2} / \mathrm{s}\right) / \mathrm{m}^{2}$ ) is given by the extinction coefficient multiplied by the flow rate through the duct divided by the exposed surface area of the sample (because cone calorimeter measurements are expressed as a function of the exposed sample surface). The integration of the SRR (or RSR) versus time curve provides the total smoke release (TSR, in $\mathrm{m}^{2}$ or $\mathrm{m}^{2} / \mathrm{m}^{2}$ ).

Smoke extinction area (SEA-in $\mathrm{m}^{2} / \mathrm{g}$ ) is calculated from cone calorimeter data as the ratio between the smoke production (in $\mathrm{m}^{2}$ ) and the mass loss (in g). The property was recorded continuously during tests, however most of the time only a mean value was reported over the whole test duration because the instantaneous value will vary significantly due to the fluctuations in the mass measurements.

It is possible to calculate smoke production as a function of the amount of released energy, and that would be expressed in $\mathrm{m}^{2} / \mathrm{kJ}$. Such calculation would relate smoke production to combustion or heat release property. Indeed, smoke production depends not only on the amount of fuel released, but also on combustion efficiency.

In this article, the rate of smoke release (RSR in $\left.\left(\mathrm{m}^{2} / \mathrm{s}\right) / \mathrm{m}^{2}\right)$ is plotted against the heat release rate in $\mathrm{kW} / \mathrm{m}^{2}$. To the best of our knowledge, this approach has never been proposed. When they studied the relations between heat release rate and smoke production rate, $\mathrm{Xu}$ et al. [11] have only considered the correlations between peaks. Four behaviors were identified from the set of materials tested (Fig. 1). Note that $\mathrm{Y}$ and $\mathrm{X}$-axis scales are different from the four figures (please refer to Figure $\mathrm{S} 1$ in supporting information to compare the four curves).

1. For some materials, the RSR/HRR ratio remains constant during the whole test (it is identified below as HRR threshold $\left(\mathrm{HRR}_{\mathrm{th}}\right)$, see below) irrespective of whether the HRR increases or decreases (labeled as type 1 materials). 

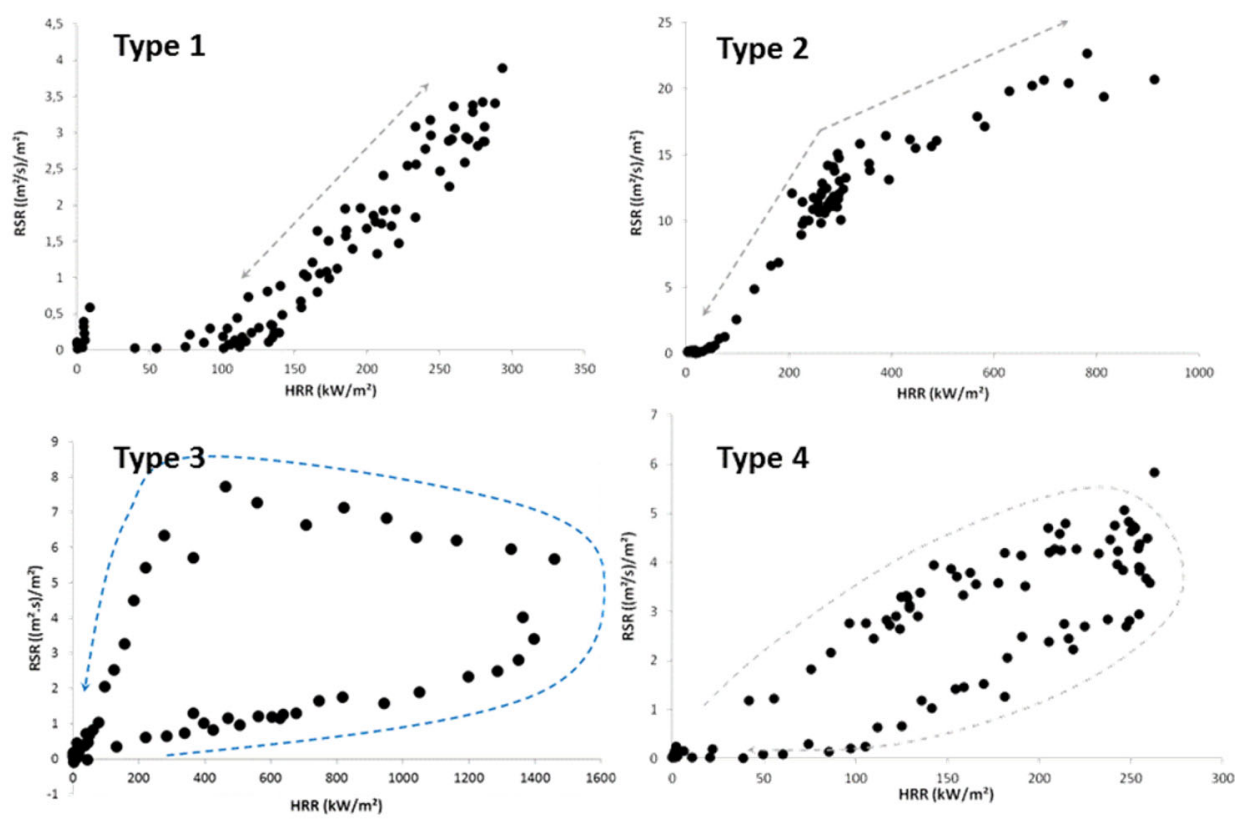

Figure 1. Various RSR versus HRR curves for different materials (type 1-PE; type 2-DGEBA-type epoxy; type 3-PA6; type 4-PE filled with $10 \mathrm{wt} \%$ of $-\mathrm{TBBPA})$.

2. For some other materials, the slope of the RSR versus HRR can change during the test but remains constant whatever the HRR increases or decreases (labeled as type 2 materials).

3. For some other materials the RSR/HRR ratio is higher following the peak HRR decrease than during the period when the HRR peak is reached (labeled as type 3 materials).

4. Finally, for some materials the RSR/HRR ratio is lower during the period following the HRR peak (labeled as type 4 materials).

The slope of the curve of RSR versus HRR corresponds to the smoke production (in $\mathrm{m}^{2}$ ) for $1 \mathrm{~kJ}$ of released energy. This slope is named A in this work (in $\left.\mathrm{m}^{2} / \mathrm{kJ}\right)$.

$$
A\left(\frac{m^{2}}{k J}\right)=\frac{R S R\left(\frac{m^{2}}{s \cdot m^{2}}\right)}{\operatorname{HRR}\left(\frac{k W}{m^{2}}\right)}
$$

By multiplying this slope by the effective heat of combustion (in $\mathrm{kJ} / \mathrm{g}$ ) over the same period of time, a new parameter is obtained named B in this work $\left(\mathrm{m}^{2} / \mathrm{g}\right)$. 


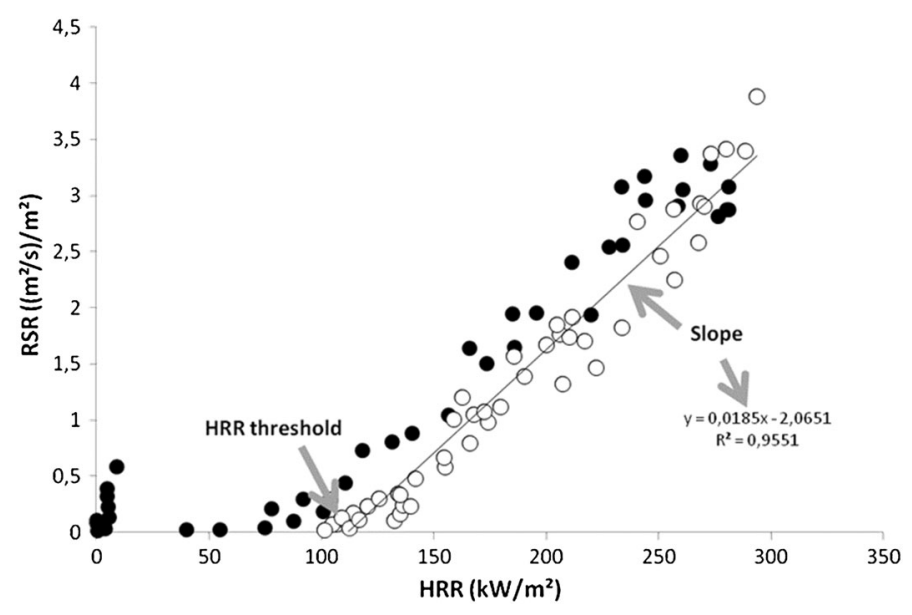

Figure 2. RSR versus HRR curve of PE with two parameters investigated in this work: slope $A$ and $H R R$ threshold $\left(H R R_{\text {th }}\right)$.

$$
B\left(\frac{m^{2}}{g}\right)=A\left(\frac{m^{2}}{k J}\right) \times E H C\left(\frac{k J}{g}\right)
$$

Another interesting parameter is called the $\mathrm{HRR}$ threshold $\left(\mathrm{HRR}_{\mathrm{th}}\right)$. Its value is the minimum heat release rate for smoke production increase (Fig. 2). A linear part of the curve can be defined by the following equation:

$$
R S R\left(\frac{m^{2}}{s \cdot m^{2}}\right)=A\left(\frac{m^{2}}{k J}\right) \times H R R\left(\frac{k W}{m^{2}}\right)+R S R_{0}\left(\frac{m^{2}}{s \cdot m^{2}}\right)
$$

$\mathrm{HRR}_{\text {th }}$ (in $\mathrm{kW} / \mathrm{m}^{2}$ ) is defined as $\frac{-R S R_{0}}{A}$ and may be negative (probably due to the inaccuracy of experimental data). This parameter can also be expressed as a mass loss rate threshold $\left(\mathrm{MLR}_{\text {th }}\right.$ in $\mathrm{g} /\left(\mathrm{s} \cdot \mathrm{m}^{2}\right)$ ) according to the following equation:

$$
\operatorname{MLR}_{t h}\left(\frac{g}{s \cdot m^{2}}\right)=\frac{H R R_{t h}\left(\frac{k W}{m^{2}}\right)}{\operatorname{EHC}\left(\frac{k J}{g}\right)}
$$

With EHC the effective heat of combustion.

Note that $\mathrm{HRR}_{\mathrm{th}}$ (or $\mathrm{MLR}_{\mathrm{th}}$ ) may be considered as a "smoke point". Indeed, it corresponds to the fuel release rate above which smoke appears. Moreover, it can be observed in Table 1 that $\mathrm{HRR}_{\text {th }}$ (or $\mathrm{MLR}_{\text {th }}$ ) is mostly positive, i.e. $\mathrm{RSR}_{0}$ is negative. Considering Eq. 3, a negative $\mathrm{RSR}_{0}$ value means that when HRR (or MLR) is multiplied by a factor X, RSR is multiplied by a factor greater than X. Then when the degradation rate increases, the smoke production is enhanced because a greater part of the fuel is not fully oxidized. This can be ascribed to the 
influence of ventilation on smoke production. Indeed, the ventilation (i.e. the ratio between oxygen and fuel) is of primary importance. When fuel production rate increases, less oxygen per fuel amount is available to oxidize soot in the flame, and smoke production increases. The parameters A and B represent how fast the smoke production increases for additional heat release (or mass loss release) above the critical value $\mathrm{HRR}_{\mathrm{th}}$ (or $\left.\mathrm{MLR}_{\mathrm{th}}\right)$.

\section{Results and Discussion}

\subsection{Preliminary Remarks}

Several remarks must be pointed out about the results calculated from the method described above. First, a proper calculation of $\mathrm{A}$ and $\mathrm{HRR}_{\mathrm{th}}$ is sometimes quite difficult when only a few points can be used for the calculation (especially in the case of fast increase in HRR to pHRR). This can explain some inaccuracies ( $\mathrm{R}^{2}$ is sometimes quite low in Table 1) and the scattering of values.

Nevertheless, standard deviation has been calculated for several polymers. From LDPE and EVA samples from series A, standard deviation calculated from 4 samples appears very satisfying for the three parameters, namely A, $H_{R} R_{\text {th }}$ and $B$ (see Table 1 in supporting information). Standard deviation was also calculated using a larger set of PMMA, PA6 and epoxy samples from series A and B. While test conditions were not exactly the same from one sample to another one, standard deviation for $\mathrm{A}$ and $\mathrm{B}$ values is higher (Table S1) but data dispersion remains acceptable (see Fig. 5 for the dispersion of B values).

Second, the ratio RSR/HRR may change continuously during the HRR plateau (around pHRR) (see for example type 4 in Fig. 1). Therefore, A and $H_{R} R_{\text {th }}$ values from the first part (HRR increase) and the final part (HRR decrease) of the curve do not allow for calculation of the exact same RSR value at pHRR.

Finally, some curves RSR $=\mathrm{f}(\mathrm{HRR})$ may be more complex as for PA6 (see type 3 in Fig. 1). Two slopes can be calculated when HRR decreases. Such a curve may provide new insights about material decomposition.

Despite these limitations, the RSR curves calculated from HRR curves and using the new parameters fit quite well with the experimental RSR curves. Figures S2 and S3 in supporting information show the calculated RSR curves for pure LDPE and EVA (using A and $H_{R}$ th values listed in Table 1) versus the experimental ones. A reasonable agreement can be observed.

\subsection{Smoke Release from Pure Polymers}

Figure 3 shows the A values versus $H_{R} R_{t h}$ for the reference series. A values correspond only to the first linear part of the curve RSR $=f(H R R)$, i.e. when HRR increases (see Fig. 2). Each point corresponds to one cone calorimeter test, therefore there are several points for one polymer in order to show the variability of calculated values. For a single polymer, the values of $A$ and $H_{R R}$ th remain close in all tests (see for example the four points corresponding to LDPE). On the con- 


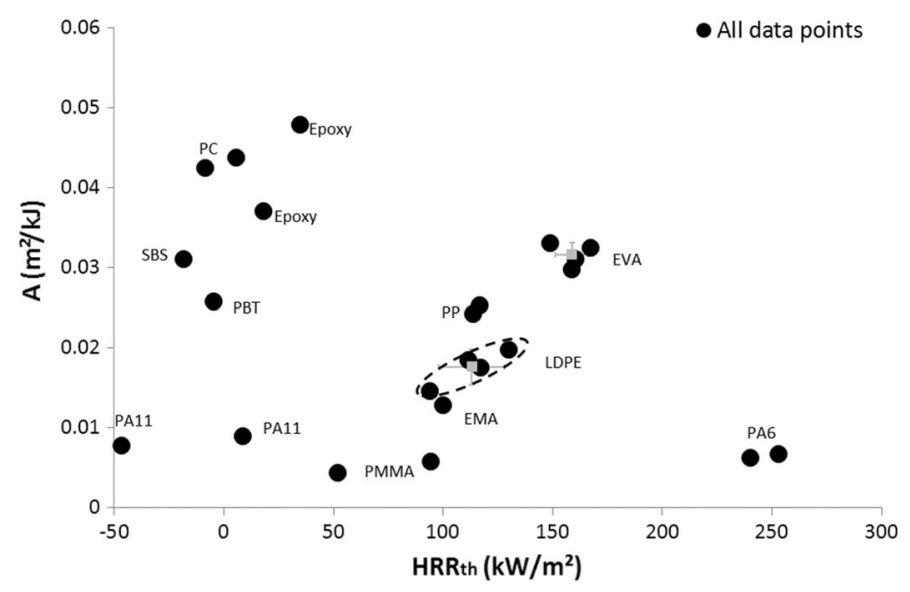

Figure 3. A $\left(\mathrm{m}^{2} / \mathrm{kJ}\right)$ versus $\mathrm{HRR}_{\mathrm{th}}$ for the polymers belonging to series A.

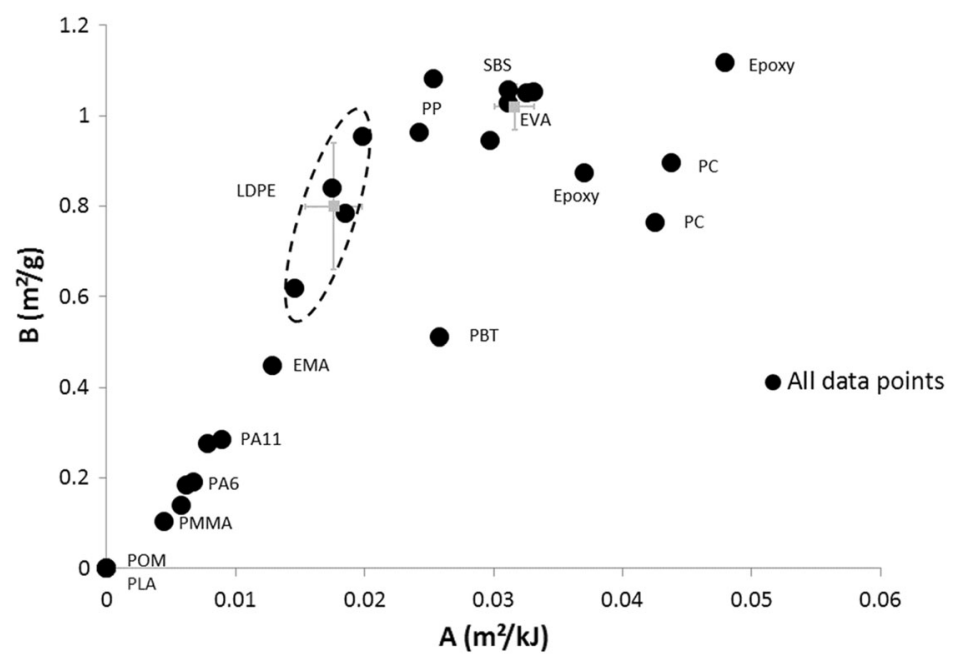

Figure 4. B $\left(\mathrm{m}^{2} / \mathrm{g}\right)$ versus $A\left(\mathrm{~m}^{2} / \mathrm{kJ}\right)$ for the polymers belonging to series A.

trary, values are significantly different between two polymers allowing to draw some conclusions.

It may be pointed out that aromatic-containing polymers exhibit rather higher A values and lower $H_{R R}$ th (close to 0). Nevertheless, the discrepancy between aliphatic and aromatic-containing polymers is not obvious. PA11 also exhibits low $\mathrm{HRR}_{\text {th }}$ and PP or EVA have A values similar to PBT or SBS.

Figure 4 shows the relation between $B$ and A values expressed in $\mathrm{m}^{2} / \mathrm{g}$ and $\mathrm{m}^{2} /$ $\mathrm{kJ}$. The ratio between both values corresponds to the effective heat of combustion 
(except for PLA and POM, for which A and B values are null). Once again, it can be observed that aromatic-containing polymers exhibit a behavior distinct from aromatic-free ones. Indeed, for similar B values in $\mathrm{m}^{2} / \mathrm{g}$, the former have higher A values in $\mathrm{m}^{2} / \mathrm{kJ}$. This means that for a similar amount of released fuels, the smoke opacity is higher and the energy released is lower for aromatic-containing polymers.

Smoke opacity is due to soot particles [12] which are formed from carbon-containing fuels. Heat release during combustion is mainly due to the oxidation of carbon groups. While all polymers studied contain only $\mathrm{C}, \mathrm{O}, \mathrm{H}$ and $\mathrm{N}$ atoms, the carbon fraction is more or less inversely proportional to the nitrogen and/or oxygen fraction. Figure 5 shows the B values versus the weight fraction of nitrogen and oxygen atoms (called $\mathrm{f}(\mathrm{N} / \mathrm{O})$ ) in the polymer structure for the reference series and the additional data (series B). Note that in most cases, polymers do not contain nitrogen atoms and $\mathrm{f}(\mathrm{N} / \mathrm{O})$ corresponds only to the weight fraction of oxygen atoms. Two observations can clearly be pointed out.

First, the $B$ values increase when $\mathrm{f}(\mathrm{N} / \mathrm{O})$ decreases. This is not surprising while soots are mainly carbon. More particularly, polymers exhibiting a high $\mathrm{f}(\mathrm{N} / \mathrm{O})$ value ( $>0.4$ ) as PLA and POM [13] do not produce smoke (or produce very little smoke). Secondly, for a similar $\mathrm{f}(\mathrm{N} / \mathrm{O})$, aromatic-containing polymers exhibit higher B values than aromatic-free ones. This implies that the presence of aromatic groups promotes the formation of soot particles and then increases smoke opacity. Once again, this is not a new result but the cone calorimeter data highlight this conclusion clearly.

Discrepancy between values is particularly obvious for the EVA results obtained from various tests. Indeed, these data were collected for various EVA tested at two different heat fluxes $\left(35\right.$ and $\left.50 \mathrm{~kW} / \mathrm{m}^{2}\right)$. It must be mentioned that

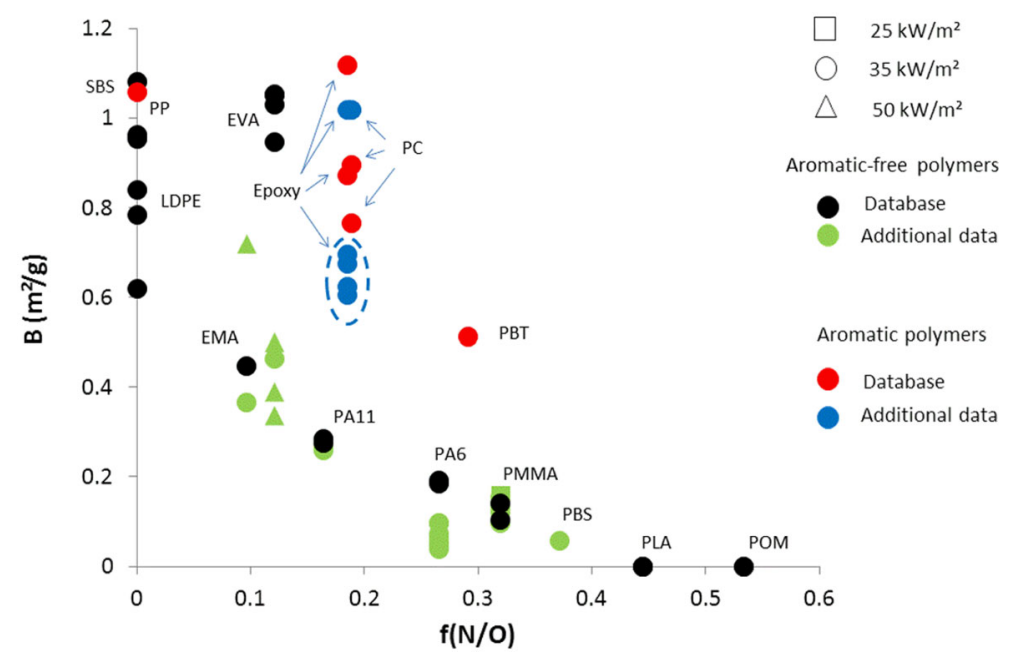

Figure 5. $B\left(\mathrm{~m}^{2} / \mathrm{g}\right)$ versus $\mathrm{f}(\mathrm{N} / O)$ weight fraction for the polymers belonging to series $A$ and $B$. 
EVA degrades in two steps and both steps release different fuels as evidenced using thermogravimetric analyses. During the first step, acetic acid (with high $\mathrm{f}(\mathrm{N} /$ $\mathrm{O})$ value) is mainly released. During the second step, polyene macromolecules (containing only $\mathrm{C}$ and $\mathrm{H}$ atoms) decompose [14]. In cone calorimeter tests, both steps are partially superimposed but the mass proportion between the fuels released from both steps changes continuously as evidenced in a previous work [15]. Moreover, this also depends on test conditions (especially heat flux), which may explain the high discrepancy between B values from one test to another.

\subsection{Smoke Suppressants}

Some mineral fillers are known to be smoke suppressants. This is especially the case for MDH [16], but in many articles, mineral fillers are considered to act as smoke suppressors on the basis of smoke production rate versus time curves. The presence of MDH or ATH in polymers obviously leads to a decrease in smoke production, due to the high content of these fillers. Another explanation is the modification of the decomposition rate of polymers in the presence of these fillers. We showed above that the RSR versus HRR curve is rather complex. A change in heat release rate (i.e. in decomposition rate) corresponds to a change in smoke production rate. However, the relation between both values is only fully linear for "type 1-polymers" with $\mathrm{HRR}_{\text {th }}$ close to 0 . In other cases, a change in heat release rate can correspond to non-proportional change in smoke production. Furthermore, MDH or ATH may also modify the smoke production through specific phenomena.

In order to identify how smoke production is reduced in the presence of fillers, series $\mathrm{C}$ was studied. Figure 6 shows the RSR versus time curves for all the for-

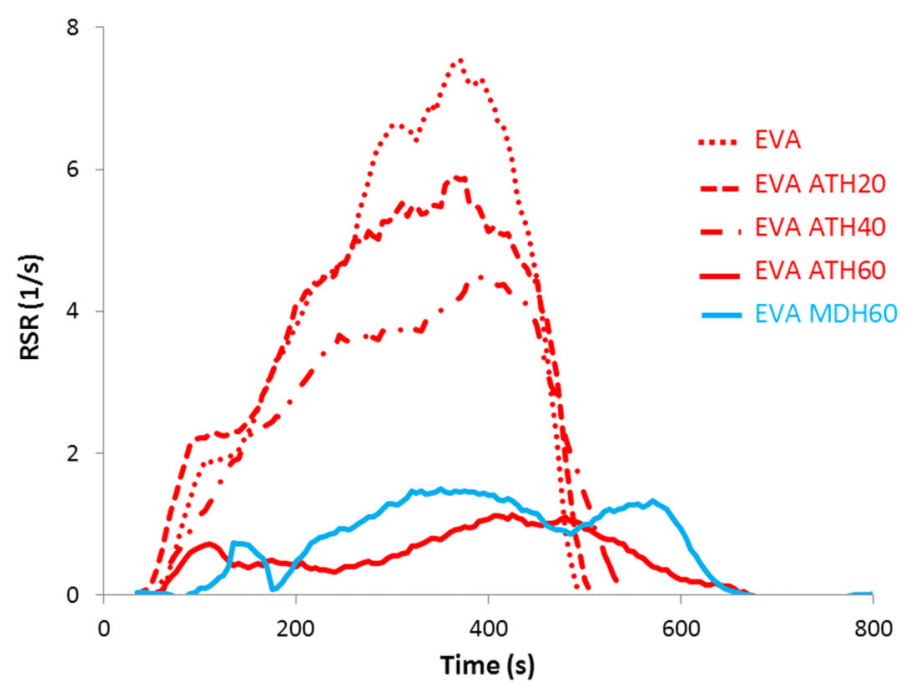

Figure 6. RSR versus time curves from cone calorimeter tests for pure EVA and EVA filled with mineral fillers. 


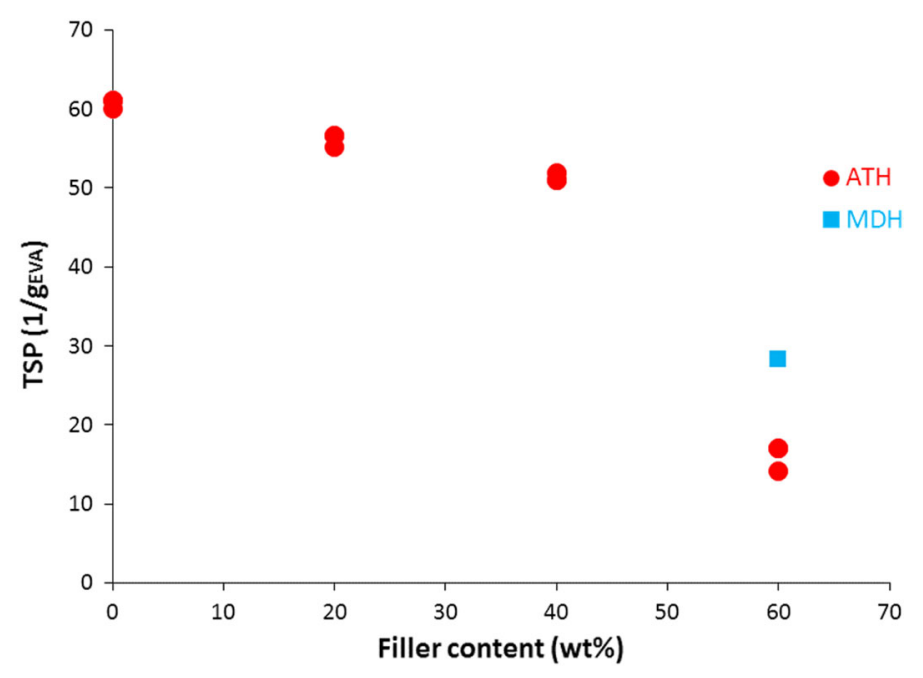
Figure 7. Total smoke release in $\mathrm{m}^{2} / \mathrm{m}^{2}$ per gram of EVA versus
filler confent for various EVA filled with ATH and MDH.

mulations from this series. Note that total smoke production (TSR - by integrating the RSR curve over time) is only $381 \mathrm{~m}^{2} / \mathrm{m}^{2}$ for EVA filled with $60 \mathrm{wt} \%$ of ATH, corresponding to only $18 \%$ of the value for pure EVA $\left(2132 \mathrm{~m}^{2} / \mathrm{m}^{2}\right)$.

Figure 7 draws the total smoke release per gram of EVA versus the filler content. While EVA is fully degraded at the end of the test, TSR $\left(\right.$ in $\left.\left(\mathrm{m}^{2} / \mathrm{m}^{2}\right) / \mathrm{g}_{\mathrm{EVA}}\right)$ should be constant if $\mathrm{MDH}$ and ATH act only through a diluting effect in condensed phase. This is not the case and a decrease in TSR can be observed, especially for $60 \mathrm{wt} \%$ of mineral fillers. The total smoke release (TSR) is only $16\left(\mathrm{~m}^{2} /\right.$ $\left.\mathrm{m}^{2}\right) / \mathrm{g}$ EVA for EVA filled with $60 \mathrm{wt} \%$ of ATH versus $61\left(\mathrm{~m}^{2} / \mathrm{m}^{2}\right) / \mathrm{g}$ EVA for pure EVA.

Figure 8 shows the TSR in $\left(\mathrm{m}^{2} / \mathrm{m}^{2}\right) / \mathrm{g}$ EvA versus the average HRR (i.e. the mean HRR calculated between ignition and flame out) for all formulations from series C. A clear linear relation can be observed, pointing out that the decrease in TSR is mainly due to the decrease in heat release rate when mineral fillers are added to EVA. It should be kept in mind that heat release rate corresponds to the decomposition rate of EVA while water released from mineral fillers does not contribute to released energy.

Nevertheless, there are also changes in the RSR versus HRR curves. Figure 9 shows the $A$ (in $\mathrm{m}^{2} / \mathrm{kJ}$ ) and $\mathrm{HRR}_{\text {th }}$ values for the different formulations when HRR increases and decreases (Fig. 9a, b, respectively). For all materials, the values are not the same when HRR increases and decreases - their behavior corresponds to type 3. Especially for the first part of the curve (when HRR increases-Fig. 9a), A and $\mathrm{HRR}_{\mathrm{th}}$ decrease in presence of fillers. When HRR decreases, $H_{R} R_{\text {th }}$ does not change significantly but $A$ value also decreases with fillers. These data suggest that ATH and MDH slightly modify the smoke production through specific phenomena to be identified, even if the decrease in smoke 


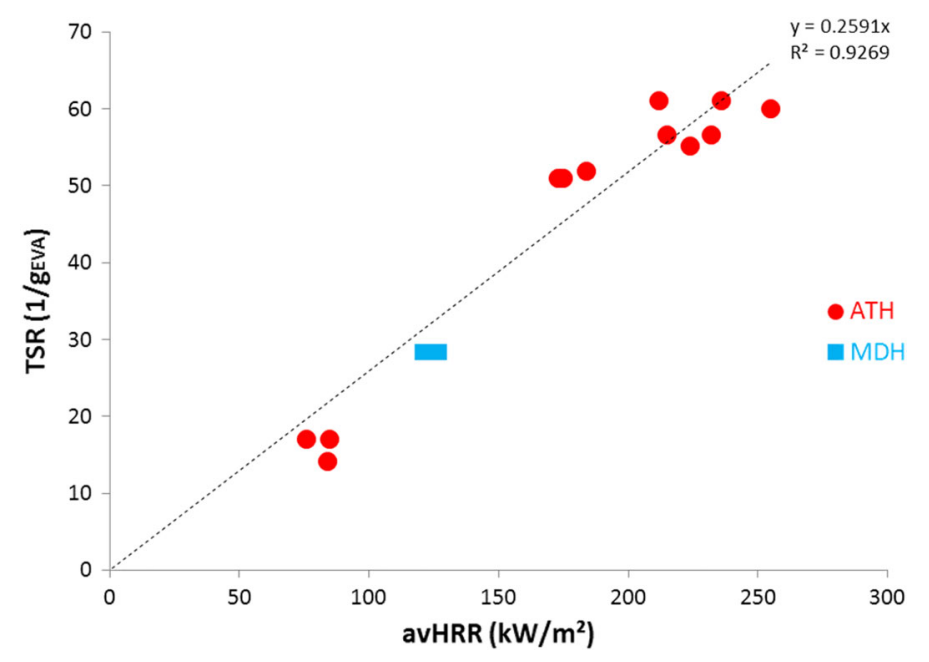

\section{Figure 8. Total smoke release in $\mathrm{m}^{2} / \mathrm{m}^{2}$ per gram of EVA versus average HRR for various EVA filled with ATH and MDH.}

production is mainly due to dilution effect and change in heat release rate, as shown previously.

\subsection{Flame Inhibitors}

Type 3 behavior is easily understood. Indeed, the smoke opacity can be promoted at the end of a cone calorimeter test when the flame starts vanishing because combustion becomes incomplete. Moreover, the char layer (containing aromatic groups) undergoes thermo-oxidation and degrades. Type 4 behavior described in Fig. 10 refers to polyethylene containing $10 \mathrm{wt} \%$ of a brominated flame retardant (TBBPA). TBBPA acts as flame inhibitor and promotes a decrease in effective heat of combustion and a release of (black) smoke [17].

The change in RSR/HRR ratio (from a higher to a lower value, as observed for type 4 behavior) is due to a change in fuel composition and combustion efficiency. Indeed, $\mathrm{HBr}$ rate was measured continuously during the cone calorimeter test. The $\mathrm{HBr}$ rate increases up to a peak value observed at $210 \mathrm{~s}$ and then decreases. The change in $\mathrm{RSR} / \mathrm{HRR}$ ratio occurs when $\mathrm{HBr}$ release becomes very low, after $6 \mathrm{~min}$. EHC also changes from $32.7 \mathrm{~kJ} / \mathrm{g}$ when $\mathrm{HRR}$ increases to $39.6 \mathrm{~kJ} / \mathrm{g}$ when HRR decreases.

Note also that at the end of the test, when $\mathrm{HBr}$ release is low, the $\mathrm{HRR}_{\mathrm{th}}$ corresponding to the second part of the curve (when HRR decreases) is close to $100 \mathrm{~kW} / \mathrm{m}^{2}$, i.e. the same value as for pure PE. Similarly A $\left(0.018 \mathrm{~m}^{2} / \mathrm{kJ}\right)$ is close to the value for pure PE (compare with Fig. 3).

DOPO is a phosphorus flame retardant well-known to act at least partially in gas phase as flame inhibitor [18]. $10 \mathrm{wt} \%$ of DOPO were added to PP and PE and compared to the same amount of TBBPA (series 3). Note that these FR are chosen as models but are not specifically used for polyolefins. 


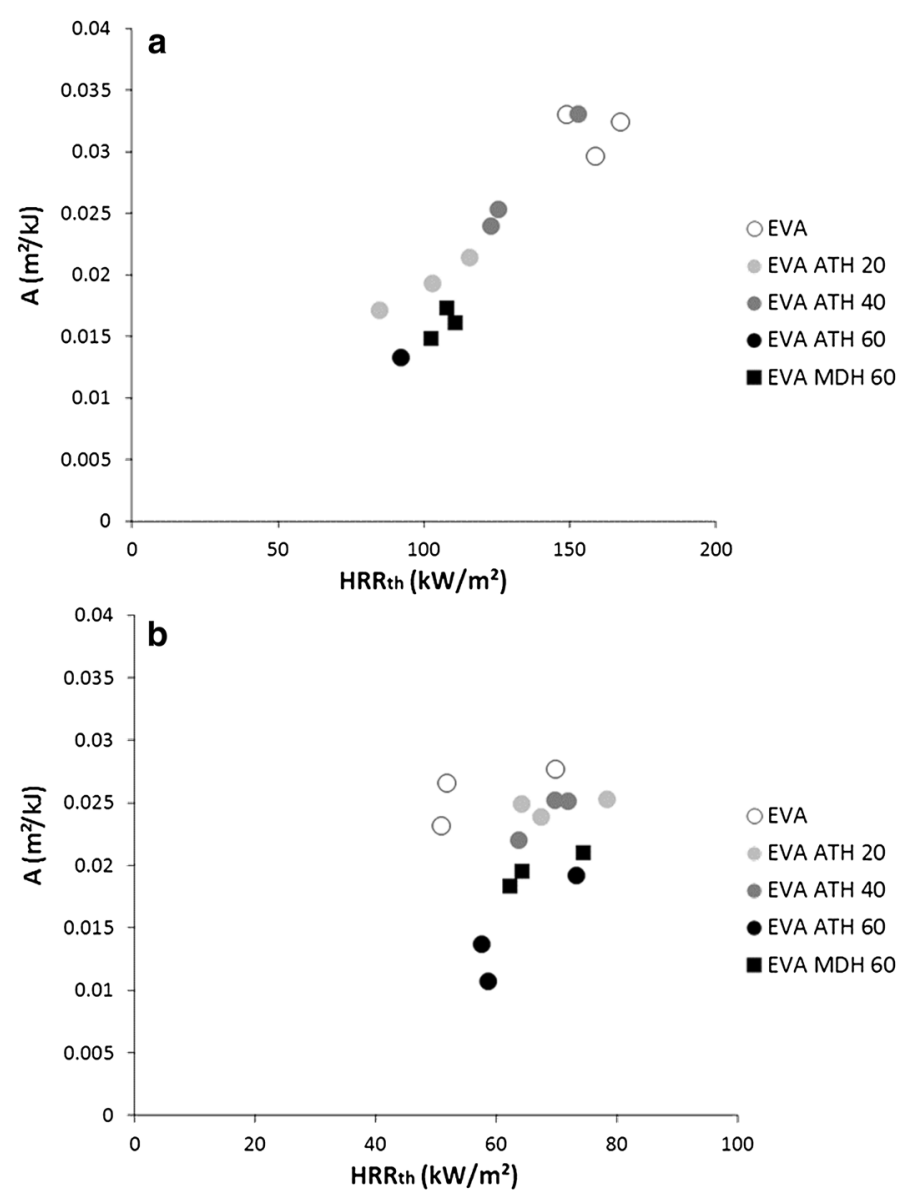

\section{Figure 9. A (in $\mathrm{m}^{2} / \mathrm{kJ}$ ) versus $\mathrm{HRR}_{\text {th }}$ for materials from series C (a HRR increasing; b HRR decreasing).}

Both flame retardants promote high smoke production: as an example, for LDPE, TSR increases from $600 \mathrm{~m}^{2} / \mathrm{m}^{2}$ to $750 \mathrm{~m}^{2} / \mathrm{m}^{2}$ for pure polymer to 1000 $1200 \mathrm{~m}^{2} / \mathrm{m}^{2}$ for LDPE with DOPO and $1200-1400 \mathrm{~m}^{2} / \mathrm{m}^{2}$ for LDPE with TBBPA. Ignition is only slightly delayed. Peak of heat release rate is decreased from $300 \mathrm{~kW} / \mathrm{m}^{2}$ for pure LDPE to $280 \mathrm{~kW} / \mathrm{m}^{2}$ for LDPE with DOPO and $260 \mathrm{~kW} / \mathrm{m}^{2}$ for LDPE with TBBPA. The time to pHRR does not change in presence of FR (around $400 \mathrm{~s}$ for PE-based materials and $250 \mathrm{~s}$ for PP-based materials).

As already discussed, PP and PE containing TBBPA exhibit a typical type 4 behavior while other materials have a type 1 behavior. Figure 11a shows the A and $\mathrm{HRR}_{\mathrm{th}}$ values for all the polymers from series $\mathrm{D}$. Note that residue content is negligible in all cases, excluding any char promotion effect. It can be observed that $\mathrm{A}$ in $\mathrm{m}^{2} / \mathrm{kJ}$ remains almost constant for a given polymer, irrespective of the 

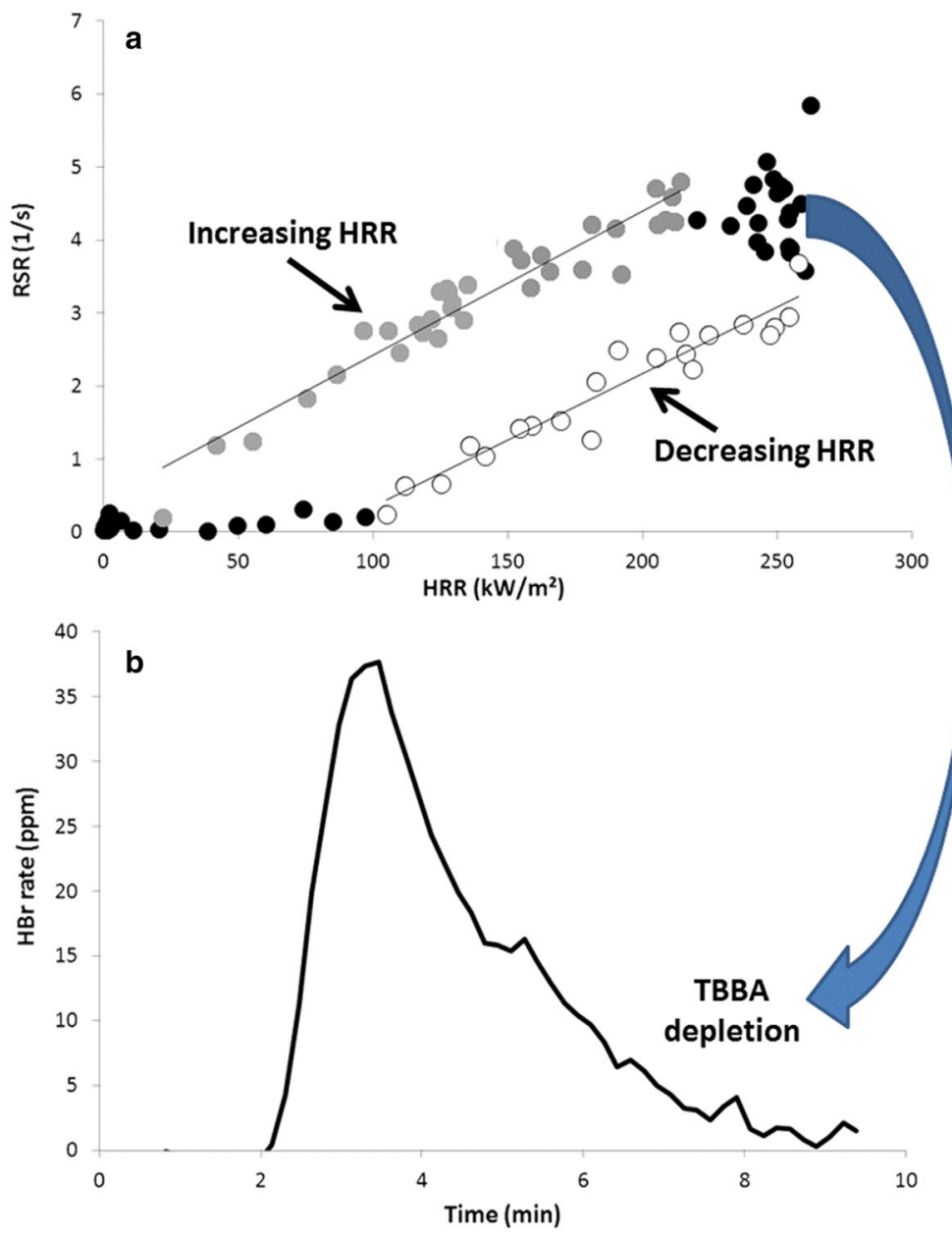

Figure 10. (a) RSR versus HRR curve and (b) HBr rate versus time for PE filled with 10 wt\% of TBBPA.

presence of FR. On the contrary, $H R R_{\text {th }}$ decreases significantly when FR is added to PP or PE. The decrease is more significant in the case of TBBPA than DOPO. Moreover, for TBBPA-containing polymers, the $H_{R R}$ th is higher when HRR decreases than when it increases (i.e. TBBPA content in released fuels decreases).

Figure $11 \mathrm{~b}$ shows the $\mathrm{HRR}_{\text {th }}$ versus EHC. It can be noted that $\mathrm{HRR}_{\text {th }}$ becomes null or even negative when HRR increases for TBBPA-filled polymers. This is related to a low effective heat of combustion. When HRR decreases, EHC as $\mathrm{HRR}_{\text {th }}$ increases. DOPO leads to a very limited decrease in EHC (around -2 or $-3 \mathrm{~kJ} / \mathrm{g}$ ) while $\mathrm{HRR}_{\text {th }}$ decreases more significantly $(-40 \%$ and $-66 \%$ respectively for PP and PE filled with DOPO). 
a
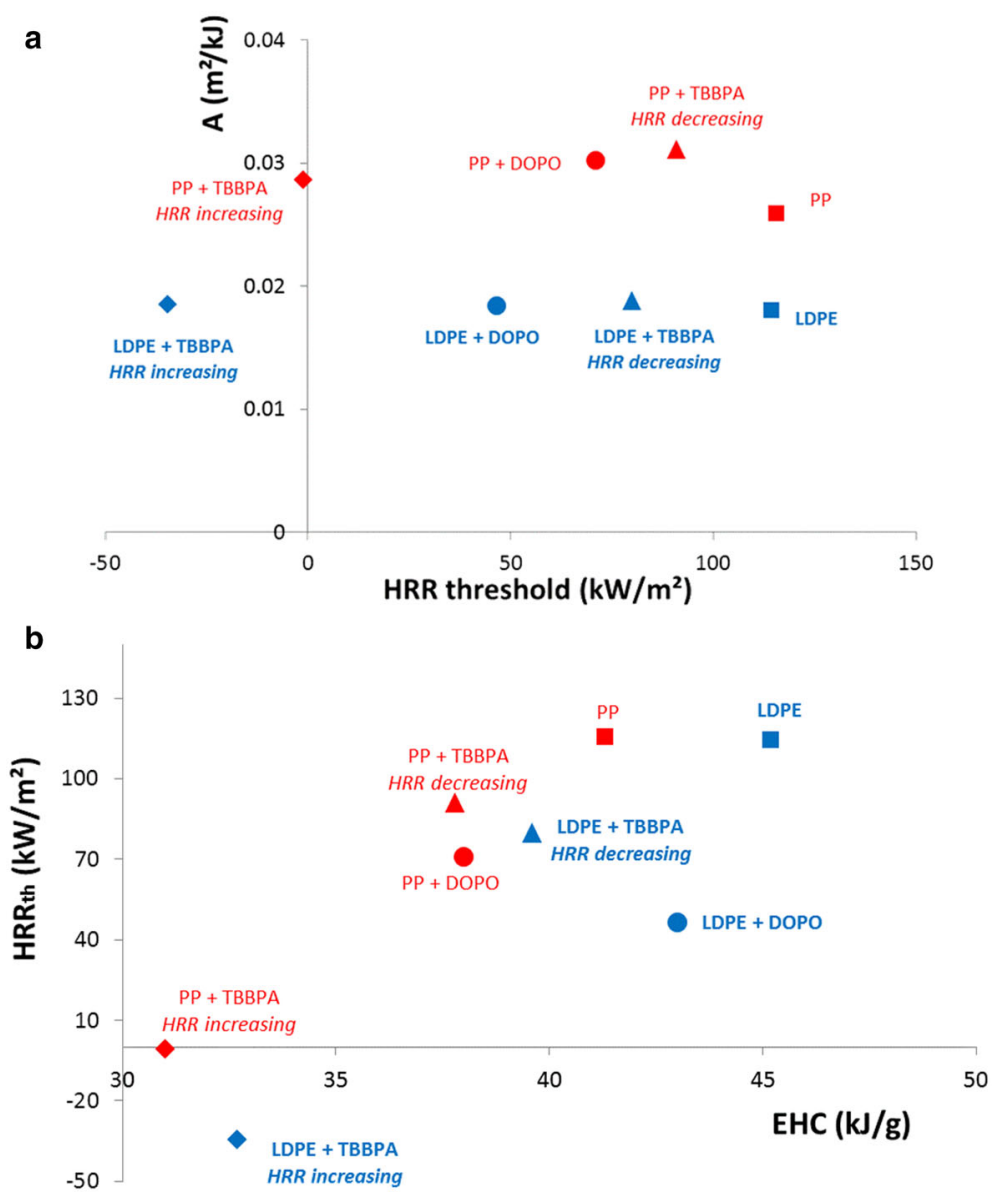

Figure 11. (a) A (in $\mathrm{m}^{2} / \mathrm{kJ}$ ) versus $\mathrm{HRR}_{\text {th }}$ and (b) $\mathrm{HRR}_{\text {th }}$ versus EHC for all the materials from series $D$.

\subsection{Comparison in Arbitrary Scenarios}

For type 3 and type 4-materials, the relation between RSR and HRR changes during the test, especially when HRR goes from an increase to a decrease. This may be ascribed to a change in released gas composition. This change is probably dependent on the decomposition rate (as evidenced for PE filled with TBBPA). On the contrary, for type 1-materials, the relation between RSR and HRR is constant and independent of the decomposition rate. Therefore, the parameters $\mathrm{A}$ and $H_{R R}$ th can be used to calculate the smoke release for an arbitrary burning scenario in order to compare different materials for the same heat release rate (or alternatively mass loss rate) curve. Obviously, this scenario still corresponds to well-ventilated combustion conditions without smoke accumulation.

This approach is carried out to compare the smoke production for PE, PP, PE filled with DOPO, PBT and SBS according to two burning scenarios, Fig. 12. The 


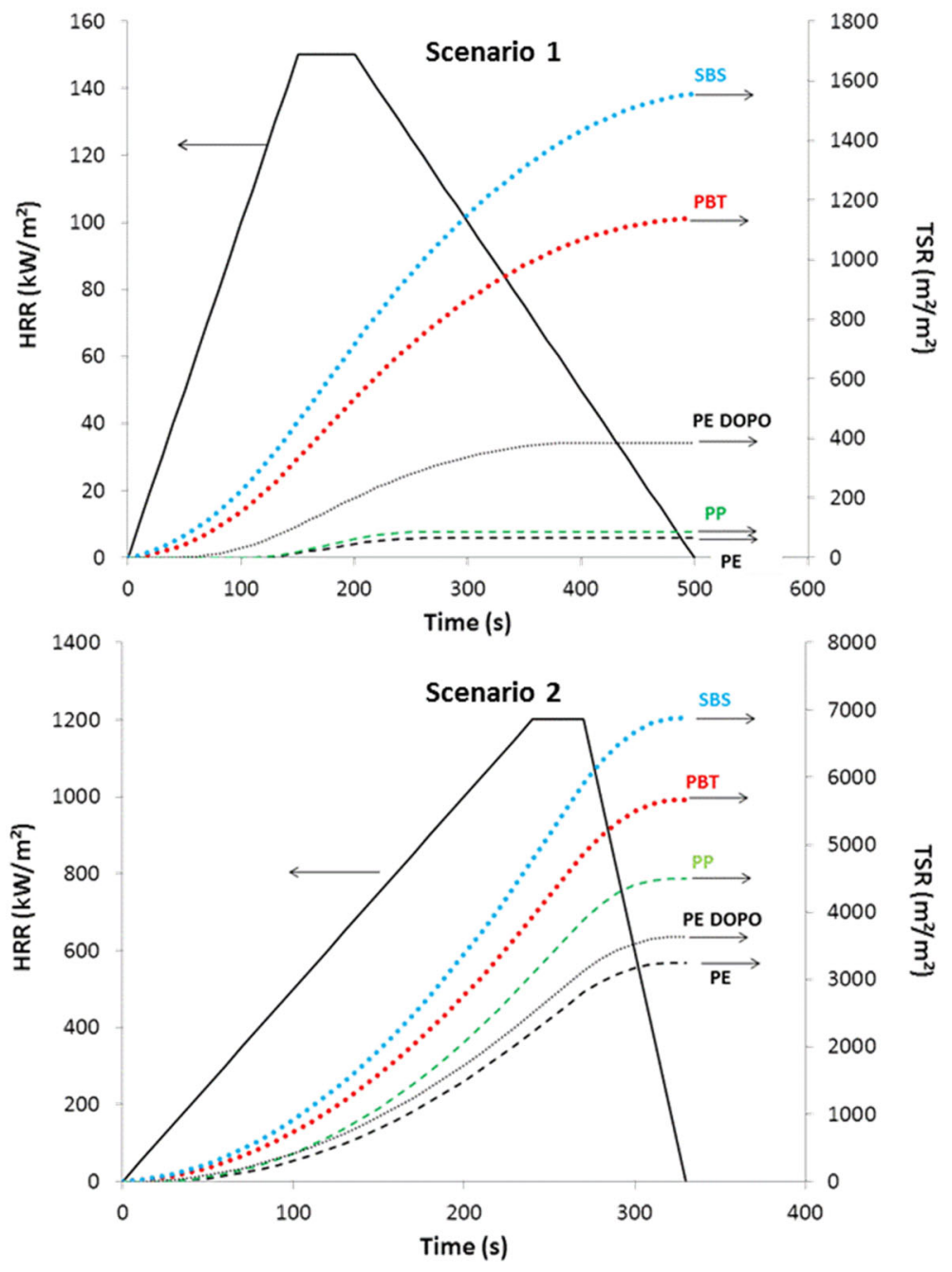

Figure 12. Total smoke release for different materials for a low burning rate scenario (scenario 1 ) and for a high burning rate scenario (scenario 2 ).

first one corresponds to a low burning rate. HRR increases up to $150 \mathrm{~kW} / \mathrm{m}^{2}$ for $150 \mathrm{~s}$, and then decreases for $300 \mathrm{~s}$ after a short plateau. The second burning scenario corresponds to a higher burning rate. HRR increases up to $1200 \mathrm{~kW} / \mathrm{m}^{2}$ for $240 \mathrm{~s}$. A short plateau is followed by a fast decrease for $60 \mathrm{~s}$.

The total smoke release for both scenarios is given in Fig. 12. When the burning rate is low (scenario 1), the TSR is very low for polyolefins compared to aromatic polymers (20 times lower, $60-80 \mathrm{~m}^{2} / \mathrm{m}^{2}$ for PE and PP versus almost $1600 \mathrm{~m}^{2} / \mathrm{m}^{2}$ for SBS). This is due to the high $\mathrm{HRR}_{\mathrm{th}}$ for PE and PP. PE filled with DOPO exhibits an intermediate smoke release value. 
When the burning rate is much higher, TSR increases significantly for all materials but the difference between polyolefins and aromatic polymers is reduced. TSR of SBS is only twice that of PE. Interestingly, PP exhibits higher TSR than PE filled with DOPO contrary to scenario 1.

These results emphasize the fact that the comparison between materials from the point of view of smoke production is fully dependent on the heat release rate, i.e. on the burning scenario.

\section{Conclusions}

Smoke release is dependent on many parameters, including ventilation (i.e. oxygen/fuel ratio which changes with fuel production rate), chemical structure of the polymer, presence of additives acting as flame inhibitor or smoke suppressant. The method developed and presented in this study aims as far as possible to differentiate between the influence of these parameters during burning in cone calorimeter tests (i.e. in well-ventilated conditions and without smoke accumulation as in smoke chamber). More generally, smoke data in cone calorimeter tests are not often analyzed in detail to elucidate the degradation mechanisms.

The new parameters calculated in this paper (namely $A$ and $H R R_{\text {th }}$ ) highlight the relationships between the smoke release rate of pure polymers, the carbon fraction and the presence of aromatic groups. Both contribute to an increase in the slope of the curve $\mathrm{RSR}=\mathrm{f}(\mathrm{HRR})$ and a decrease in $\mathrm{HRR}_{\mathrm{th}}$.

There is a need to assess the benefits of flame retardants by considering both their effect on heat release rate and smoke production rate. The study presented here is an attempt to address this challenge by providing a comparison between flame inhibitors according to their efficiency to reduce combustion and to promote smoke. The method also helps to identify the influence of some additives on smoke production. As an example, it has been shown that ATH and MDH decrease smoke release mainly by diluting the condensed phase fuel and by reducing heat release rate. Their contribution through another specific smoke suppressant effect is minor.

Finally, in certain cases, it becomes possible to calculate the smoke release of a material for an arbitrary heat release scenario. This would also make the comparison between various flame retardant materials easier.

As already mentioned our approach does contain some limitations and refers especially to well-ventilated fires.

\section{Acknowledgements}

The authors thank Benjamin Gallard and Loïc Dumazert for their help to prepare and characterize the formulations and Marcelo Hirschler for his review of a preliminary version. The authors also thank the group "Dégradation thermique et comportement au feu des matériaux organiques" of the Société Chimique de France to fund a part of this work. 


\section{ELECTRONIC SUPPLEMENTARY MATERIAL}

The online version of this article (https://doi.org/10.1007/s10694-018-0806-z) contains supplementary material, which is available to authorized users.

\section{References}

1. Hall JR Jr (2011) Fatal effects of fire, report, 2011. National Fire Protection Association

2. Alarifi AA, Phylaktou HN, Andrews GE (2016) What kills people in a fire? Heat or smoke? In: Presented at the 9 th Saudi students conference, 13-14 February 2016, ICC. University of Birmingham, Birmingham

3. Milke JA (2000) Evaluating the smoke hazard from fires in large spaces. Int $\mathbf{J}$ Eng Perform Based Fire Codes 2(3):94-103

4. Gyppaz F (2014) Smoke and safety in case of fire. https://www.nexans.com/Corporate/ 2014/EN-White_paper_smoke.pdf

5. Levchik S, Hirschler M, Weil E (2011) Regulations, codes and standards associated with smoke. In: Practical guide to smoke and combustion products from burning polymers: generation, assessment and control. Imprint, Shropshire

6. Baxter CS (2012) Smoke and combustion products, Ph.D., First published: 17 August 2012. https://doi.org/10.1002/0471435139.tox108.pub2

7. De Ris J, Cheng X-F (1994) The role of smoke-point in material flammability testing, fire safety journal. Proc Fourth Int Symp 4:301-312

8. Linteris GT, Rafferty IP (2008) Flame size, heat release, and smoke points in materials flammability. Fire Saf J 43:442-450

9. Levchik S, Hirschler M, Weil E (2011) Fire tests to assess smoke and combustion-product generation. In: Practical guide to smoke and combustion products from burning polymers: generation, assessment and control. Imprint, Shropshire

10. Wu X, Wang L, Wu C, Yu J, Xie L, Wang G, Jiang P (2012) Influence of char residues on flammability of EVA/EG, EVA/NG and EVA/GO composites. Polym Degrad Stab 97:54-63

11. Xu Z, Yan L, Liu Y (2014) Study on correlations between the flammability and dynamic smoke properties of four decorative materials. Proc Eng 84:498-505

12. Roessler DM, Faxvog FR (1979) Opacity of black smoke: calculated variation with particle size and refractive index. Appl Opt 18:1399-1403

13. Beaulieu P, Dembsey N (2008) Effect of oxygen on flame heat flux in horizontal and vertical orientations. Fire Saf J 43:410-428

14. Costache MC, Jiang DD, Wilkie CA (2005) Thermal degradation of ethylene-vinyl acetate coplymer nanocomposites. Polymer 46:6947-6958

15. Sonnier R, Viretto A, Dumazert L, Gallard B (2016) A method to study the two-step decomposition of binary blends in cone calorimeter. Combust Flame 169:1-10

16. White S (1998) Smoke suppressants. In: Pritchard G (ed) Plastics additives: an A-Z reference Springer, Netherlands, Dordrecht, pp 576-583

17. Visakh PM (2015) Advances in flame retardant of different types of nanocomposites. In: Visakh PM, Arao Y (eds) Flame retardants: polymer blends, composites and nanocomposites Springer, Cham, pp 1-13 
18. König A, Kroke E (2012) Flame retardancy working mechanism of methyl-DOPO and MPPP in flexible polyurethane foam. Fire Mater 36:1-15 